\title{
New Approach for the Detection of Family of Geometric Shapes in the Islamic Geometric Patterns
}

\author{
Ait Lahcen Yassine ${ }^{1}$, Jali Abdelaziz ${ }^{2}$, El Oirrak Ahmed ${ }^{3}$, Abdelmalek. Thalal ${ }^{4}$ \\ Youssef. Aboufadil ${ }^{5}$, M. A. Elidrissi $\mathrm{R}^{6}$ \\ Laboratory of Material Sciences, The University of Cadi Ayyad, Faculty of Sciences Semlalia, Marrakech, Morocco $1,2,4,5,6$ \\ Laboratory of engineering information system, The University of Cadi Ayyad ${ }^{3}$ \\ Faculty of Sciences Semlalia, Marrakech, Morocco ${ }^{3}$
}

\begin{abstract}
This article proposes a new approach to detect the family of geometric shapes in Islamic geometric patterns. This type of geometric pattern which is constructed by tracing the grids with the respect of precise measurement criteria and the concept of symmetry of a method which is called 'Hasba'. This geometric pattern generally found in the tiles which cover the floors or walls of many buildings around the Islamic world such as mosques. this article describe a new method which is based on the calculation of the Euclidean distance between the different geometric shapes which constitute the geometric Islamic pattern, in order to detect similar regions in this type of geometric pattern encountered in Islamic art.
\end{abstract}

Keywords-Family geometric; shapes; Euclidean distance; 'Hasba'; geometric art; Islamic patterns

\section{INTRODUCTION}

Since centuries, the Islamic world has had great decorative traditions. The Islamic geometric patterns [1] were very widespread throughout different countries in Africa, Asia and Europe. This article focus on the ornamental Arabesque (Thalal $\&$ al. [1-2]). More precisely, in this paper will be interested on the geometric patterns called "Tastyr" (Fig. 1). This kind of Islamic patterns are built through a method called 'Hasba' based on the respect of some precise criteria of measurement.

In Morocco, the method to build the geometric patterns adopted by craftsmen is called 'Hasba' [2], based on rigorous geometric rules such as the distance between two close neighboring geometric shapes must be fixed. The concept of 'symmetry', also based on a specific measure, is rather adapted in the carving and painting of wood, metal and plaster.

The main goal of the proposed approach consists of detecting family of similar regions through the analysis of pictures constructed based on the method 'Hasba', which containing geometric shapes. This method called "Hasba" (measure) is widely adopted by the Moroccan craftsmen ("Maâlam") especially who's working on wood material and handed over to their disciples.

This new approach to extract similar regions in Islamic geometric patterns is based on the detection first of the outline [3] of geometric shapes. In a second step, calculating the Euclidean distance [4] between them, in order to help artisans to make a decision very quickly on the geometric pattern, does it respect the rules of the 'Hasba' method ? as for example the distance between two neighboring forms is constant and the concept of symmetry is also respected.

After that the "Maâlam" can adapted the geometric pattern on wood or in other material (plaster, metal, marble...). To do so, the method of simple blob detector [5-11] is adapted. This method of having the different regions in a picture has in the input an image with a grid level [12]. The output is a set of regions.

The paper is organized as follows: In Section 2, related work. In Section 3, describe the method simple blob detector. In Section 4, shows the procedure to follow to apply the proposed approach. Section 5 contains the conclusion and the future works.

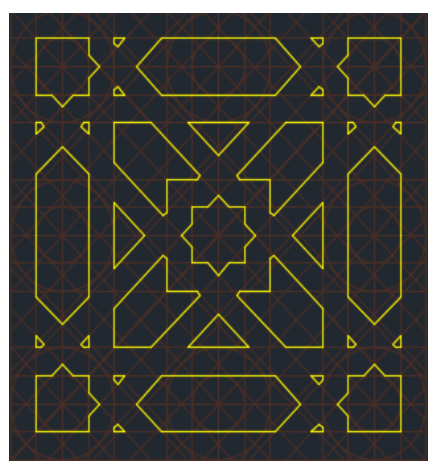

Fig. 1. Geometric Islamic Pattern.

\section{RELATED WORK}

We have find articles that talk about the Hasba method, which use some software to create the models respecting 'Hasba' method. But none articles talk about how to solve the difficulty of the validation of a geometric pattern adapted by craftsmen by respected the concept of symmetry and the new Islamic geometric pattern witch take on consideration the rules of 'Hasba' method. This article talk about a new approach that based on given the steps to follow in order to give the "Maâlam" the possibility to take the decision of the geometric pattern is valid to adapt in a wood or not.

\section{Simple Blob Detector}

To determine the different geometric shapes, this method [13-17] uses a binary picture that allows extracting the different regions that make up the picture represent the geometric pattern. The method can follow two ways of 
extracting the different regions either by using 4-connectivity as shown in Fig. 2 or 8-connectivity as shown in Fig. 3.

\section{A. Connectivity-4}

This way of traversing a picture to find the different contours of the geometric shapes in the picture makes it possible to determine the corresponding neighboring pixels.

The connectivity-4 is based on the approach by flood fill that takes a binary picture in the input as shown in Fig. 4. Which represent a binary picture [18] where the value 'true' represents ' 1 ' and the value 'false' represents 0 . It gives the different shapes in the output in the form of a matrix of labels as shown in Fig. 5.

In Fig. 5, the different geometric shapes in the picture represented with numeric values 0,1 and 2 . This means that the picture represent the geometric pattern, contains three regions.

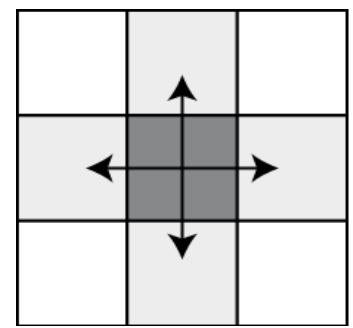

Fig. 2. Connectivity-4.

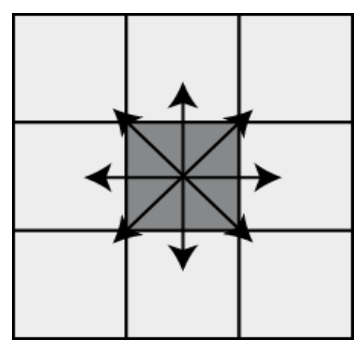

Fig. 3. Connectivity-8.

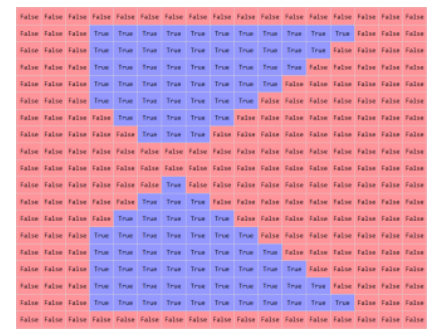

Fig. 4. Binary Picture (True (1) or False (0).

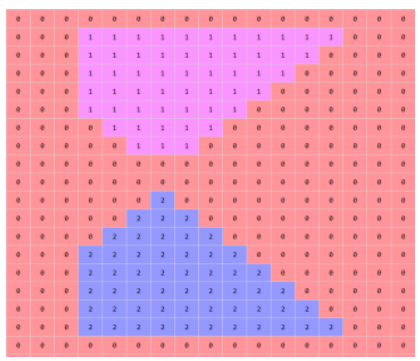

Fig. 5. Numeric Labels of each Regions (0, 1 and 2).
1) Algorithm of the approach by flood fill: The approach by flood fill based on two matrices, the first one representing the binary picture. It can be called A. The second Matrix represents the different regions founded in the picture, called B. The main algorithm Fig. 6.

\section{B. Connectivity-8}

The approach by double course that takes the binary picture in the input and it gives as output the different regions in the form of a matrix of labels as shown in Fig. 5. This second principle scan order of raster picture based on the classical sense as shown in Fig. 6. In addition, the second course is in the opposite direction.

1) Algorithm of the approach double course: The approach double course takes two matrices, the first one represents the binary picture previously named $\mathrm{A}$. The second one represents the regions already named $B$. The main algorithm used in this approach to extract the geometric shapes as shown in Fig. 7.

\section{Results}

The identification of the regions, using the Simple Blob Detector method, takes in the input (see Fig. 8) a picture in grayscale and gives in the output the two shapes in the original picture (see Fig. 9 and Fig. 10).

The different shapes with basic proprieties $\left(x_{1}^{i}, y_{1}^{i}\right)$, which represent the coordinate's point of the top left corner, $\left(x_{2}^{i}, y_{2}^{i}\right)$ represent the coordinates of the point in the bottom right corner, for the Shape ${ }^{i}$ which i represent the number of the first shape as shown in Fig. 9.

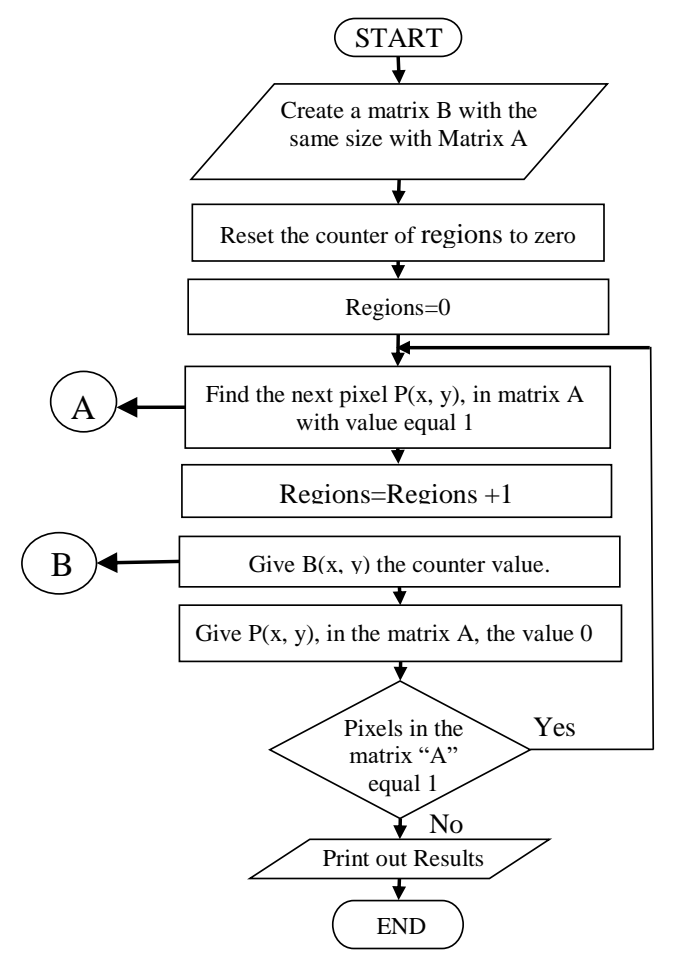

Fig. 6. Main Flow Chart of the Algorithm used in this Approach by Flood Fill with Connectivity-4. 


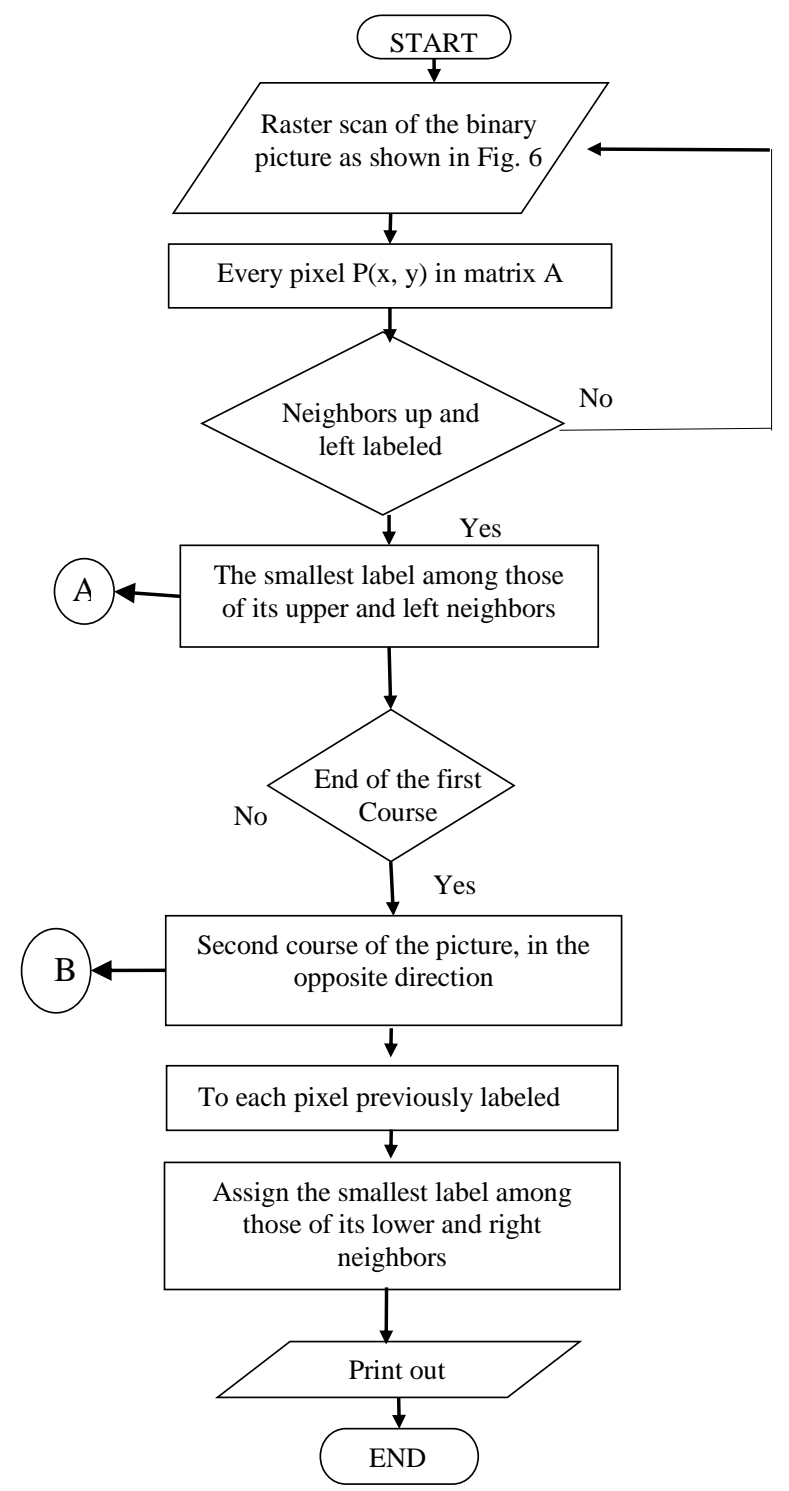

Fig. 7. Main Flow Chart of the Algorithm used in this Approach by Flood Fill with Connectivity-8.

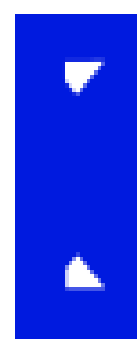

Fig. 8. Input Picture.

$\left(x_{1}^{j}, y_{1}^{j}\right)$ represent the coordinates of the point in the top left corner, $\left(x_{2}^{j}, y_{2}^{j}\right)$ represent the coordinates of the point in the bottom right for the Shape ${ }^{j}$ which $\mathrm{j}$ represent the number of the second shape as shown in Fig. 10.

In Table I, presented the $\mathrm{x}$ and $\mathrm{y}$ coordinates of the top left corner and the bottom right one for the two shapes, as shown in Fig. 11.

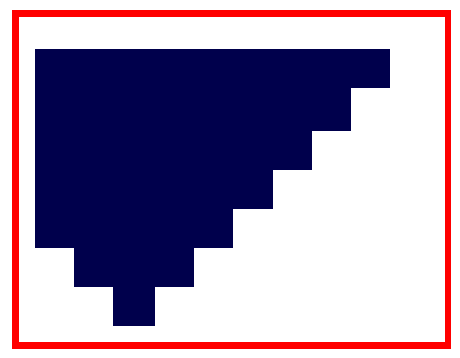

Fig. 9. Shape 1 Detected from Original Image.

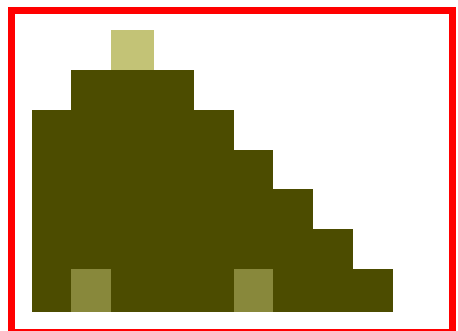

Fig. 10. Shape 2 Detected from Original Image.

TABLE. I. COORDINATES X AND Y FOR SHAPE

\begin{tabular}{|l|l|l|}
\hline \multirow{2}{*}{ Coordinates } & Shape & shape \\
\cline { 2 - 3 } & shape $^{i}$ & 11 \\
\hline Coordinates $\mathrm{x}_{1}$ & 2 & 18 \\
\hline Coordinates $\mathrm{y}_{1}$ & 8 & 19 \\
\hline Coordinates $\mathrm{x}_{2}$ & 9 & 19 \\
\hline Coordinates $\mathrm{y}_{2}$ & 19 & \\
\hline
\end{tabular}

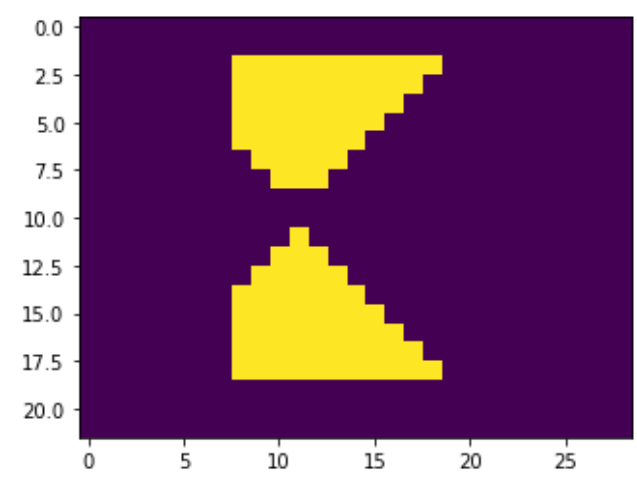

Fig. 11. Basic Proprieties $\left(\boldsymbol{x}_{1}, \boldsymbol{y}_{1}, \boldsymbol{x}_{2}, \boldsymbol{y}_{2}\right)$ for each Shapes.

\section{PROPOSED APPROACH}

In this work, the Simple Blob Detector method is used with connectivity-8 to extract the different regions [8-9] in a Hasba picture Fig. 12. In order to solve the validation problem in this kind of Islamic geometric pattern, which is the distance between two neighboring geometric shapes must be the same.

The proposed approach is focus on the first part of the problem, which is how to extract similar shapes composing a family, as shown in Fig. 13.

Now, the experiment test of the approach based on detecting the different geometric shapes in 'Hasba' picture, and compute the Euclidian distance between every couple of shapes Fig. 14 and Fig. 15. 


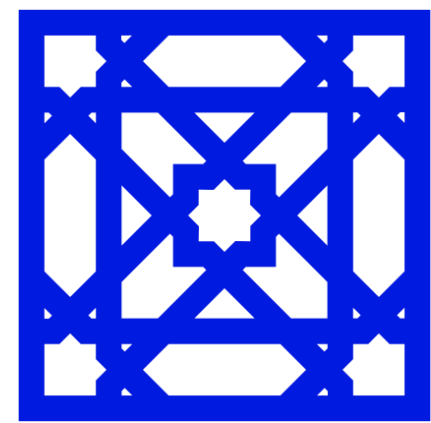

Fig. 12. Input Image Hasba.

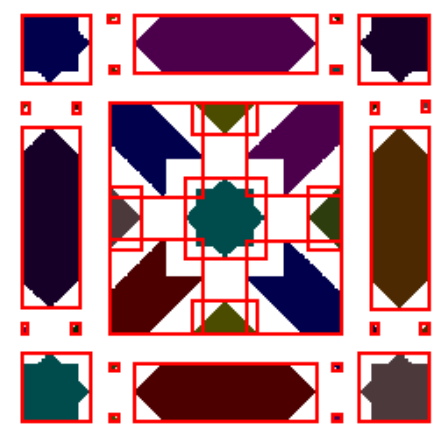

Fig. 13. Geometric Shapes Detected.

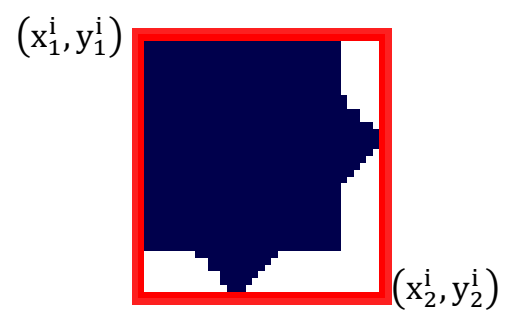

Fig. 14. Geometric Shape Characterized by $\left(\mathrm{x}_{1}, \mathrm{y}_{1}\right)$ and $\left(\mathrm{x}_{2}, \mathrm{y}_{2}\right)$.

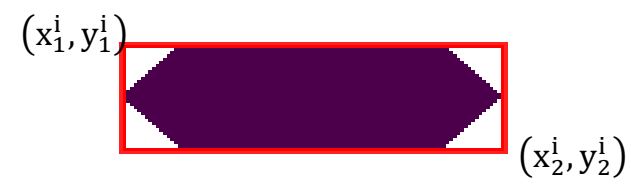

Fig. 15. Geometric Shape Characterized by (x1,y1) and (x2, y2).

\section{A. Similarity between Regions}

In Table II, represent the coordinates $\left(\mathrm{x}_{1}, \mathrm{y}_{1}\right)$ and $\left(\mathrm{x}_{2}, \mathrm{y}_{2}\right)$ of the different points for the every shape ${ }^{\mathrm{i}}$ detected in the 'Hasba' picture with the Simple blob detector method.

After having detected the different coordinates $\mathrm{x}$ and $\mathrm{y}$ for each shape in the Islamic geometric pattern. The next step is to compute the Euclidian distance, using Eq. (1), between the shape ${ }^{\mathrm{i}}$, with coordinates $\left[\left(x_{1}^{i}, y_{1}^{i}\right),\left(x_{2}^{i}, y_{2}^{i}\right)\right]$ and the shape ${ }^{\mathrm{j}}$ with coordinates $\left[\left(x_{1}^{j}, y_{1}^{j}\right),\left(x_{2}^{j}, y_{2}^{j}\right)\right]$ where $(\mathrm{i}, \mathrm{j}) \in[1, \mathrm{n}]$ and $\mathrm{n}$ represent the number of shapes in the Islamic geometric pattern.

$D_{i, j}^{1}=\sqrt{\left(x_{1}^{i}-x_{1}^{j}\right)^{2}+\left(y_{1}^{i}-y_{1}^{j}\right)^{2}}$
In addition, the second distance using Eq. (2).

$D_{i, j}^{2}=\sqrt{\left(\mathrm{x}_{2}^{\mathrm{i}}-\mathrm{x}_{2}^{\mathrm{j}}\right)^{2}+\left(\mathrm{y}_{2}^{\mathrm{i}}-\mathrm{y}_{2}^{\mathrm{j}}\right)^{2}}$

1) Proposed Distances used to compare the different shapes

If ${ }_{i, j}^{1} D \cong{ }_{i, j}^{2} D$, that means the two shapes are not necessarily similar.

After some tests, founded that there was no equality between the two distances $\left[D_{i, j}^{1}, D_{i, j}^{2}\right]$ and the two shapes [shape ${ }^{i}$, shape $\left.{ }^{j}\right]$ are similar. As shown in Table III.

TABLE. II. COORDINATES $\left(\mathrm{X}_{1}, \mathrm{Y}_{1}\right)$ AND $\left(\mathrm{X}_{2}, \mathrm{Y}_{2}\right)$

\begin{tabular}{|c|c|c|c|c|}
\hline \multirow{2}{*}{ shapes $^{i}$} & \multicolumn{4}{|c|}{ Coordinates } \\
\hline & $x_{1}^{i}$ & $y_{1}^{i}$ & $x_{2}^{i}$ & $y_{2}^{i}$ \\
\hline Shape 1 & 27 & 27 & 89 & 89 \\
\hline Shape 2 & 27 & 105 & 33 & 114 \\
\hline Shape 3 & 27 & 128 & 79 & 292 \\
\hline Shape 4 & 27 & 305 & 34 & 314 \\
\hline Shape 5 & 27 & 331 & 89 & 393 \\
\hline Shape 6 & 73 & 106 & 79 & 114 \\
\hline Shape 7 & 73 & 305 & 79 & 314 \\
\hline Shape 8 & 105 & 387 & 114 & 393 \\
\hline Shape 9 & 106 & 27 & 115 & 34 \\
\hline Shape 10 & 106 & 73 & 115 & 79 \\
\hline Shape 11 & 106 & 106 & 191 & 190 \\
\hline Shape 12 & 106 & 181 & 135 & 239 \\
\hline Shape 13 & 106 & 229 & 190 & 314 \\
\hline Shape 14 & 106 & 341 & 115 & 347 \\
\hline Shape 15 & 128 & 27 & 291 & 79 \\
\hline Shape 16 & 128 & 341 & 292 & 393 \\
\hline Shape 17 & 174 & 174 & 246 & 246 \\
\hline Shape 18 & 181 & 106 & 239 & 135 \\
\hline Shape 19 & 181 & 285 & 239 & 314 \\
\hline Shape 20 & 229 & 106 & 314 & 190 \\
\hline Shape 21 & 230 & 230 & 314 & 314 \\
\hline Shape 22 & 285 & 181 & 314 & 239 \\
\hline Shape 23 & 305 & 27 & 314 & 33 \\
\hline Shape 24 & 305 & 72 & 314 & 79 \\
\hline Shape 25 & 305 & 341 & 314 & 347 \\
\hline Shape 26 & 305 & 386 & 314 & 393 \\
\hline Shape 27 & 331 & 27 & 393 & 89 \\
\hline Shape 28 & 331 & 331 & 393 & 393 \\
\hline Shape 29 & 341 & 106 & 347 & 115 \\
\hline Shape 30 & 341 & 128 & 393 & 292 \\
\hline Shape 31 & 341 & 306 & 347 & 314 \\
\hline Shape 32 & 386 & 106 & 393 & 115 \\
\hline Shape 33 & 387 & 306 & 393 & 315 \\
\hline
\end{tabular}


TABLE. III. List OF THE Distances $D_{i, j}^{1}$ AND $D_{i, j}^{2}$ FOR THE ShaPeS

\begin{tabular}{|c|c|c|c|c|}
\hline \multirow{2}{*}{ shapes } & \multicolumn{4}{|c|}{ Distances $D_{i, j}^{1}$ and $D_{i, j}^{2}$} \\
\hline & Shape ${ }^{i}$ & Shape $^{j}$ & $D_{i, j}^{1}$ & $D_{i, j}^{2}$ \\
\hline Shape 1 & 1 & 1 & 0 & 0 \\
\hline Shape 2 & 1 & 5 & 304.0 & 304.0 \\
\hline Shape 3 & 1 & 27 & 304.0 & 304.0 \\
\hline Shape 4 & 1 & 28 & 429.92 & 429.92 \\
\hline Shape 5 & 2 & 2 & 0 & 0 \\
\hline Shape 6 & 2 & 4 & 200.0 & 200.0 \\
\hline Shape 7 & 2 & 6 & 46.01 & 46.01 \\
\hline Shape 8 & 2 & 7 & 205.22 & 205.22 \\
\hline Shape 9 & 2 & 8 & 292.58 & 290.52 \\
\hline Shape 10 & 2 & 9 & 111.01 & 114.56 \\
\hline Shape 11 & 2 & 10 & 85.2 & 89.15 \\
\hline Shape 12 & 2 & 14 & 248.87 & 247.0 \\
\hline Shape 13 & 2 & 23 & 288.73 & 292.44 \\
\hline Shape 14 & 2 & 24 & 279.95 & 283.1 \\
\hline Shape 15 & 2 & 25 & 364.66 & 365.0 \\
\hline Shape 16 & 2 & 26 & 395.27 & 395.98 \\
\hline Shape 17 & 2 & 29 & 314.00 & 314.00 \\
\hline Shape 18 & 2 & 31 & 372.82 & 372.28 \\
\hline Shape 19 & 2 & 32 & 359.0 & 360.0 \\
\hline Shape 20 & 2 & 33 & 412.3 & 412.3 \\
\hline Shape 21 & 3 & 3 & 0 & 0 \\
\hline Shape 22 & 3 & 15 & 142.83 & 300.5 \\
\hline Shape 23 & 3 & 16 & 235.73 & 235.73 \\
\hline Shape 24 & 3 & 30 & 314.0 & 314.0 \\
\hline Shape 25 & 11 & 11 & 0 & 0 \\
\hline Shape 26 & 11 & 13 & 123.0 & 124 \\
\hline Shape 27 & 11 & 20 & 123.0 & 123.0 \\
\hline Shape 28 & 11 & 21 & 175.36 & 174.65 \\
\hline Shape 29 & 12 & 12 & 0 & 0 \\
\hline Shape 30 & 12 & 18 & 106.06 & 147.07 \\
\hline Shape 31 & 12 & 19 & 128.22 & 128.22 \\
\hline Shape 32 & 12 & 22 & 179.0 & 179.0 \\
\hline Shape 33 & 17 & 17 & 0 & 0 \\
\hline
\end{tabular}

To solve this problem, the distances in Eq. (3) and Eq. (4) present in Table IV are added to solve the problem detected.

$D_{i, j}^{3}=\sqrt{\left(\mathrm{x}_{1}^{\mathrm{i}}-\mathrm{x}_{2}^{\mathrm{j}}\right)^{2}+\left(\mathrm{y}_{1}^{\mathrm{i}}-\mathrm{y}_{2}^{\mathrm{j}}\right)^{2}}$

$D_{i, j}^{4}=\sqrt{\left(\mathrm{x}_{2}^{\mathrm{i}}-\mathrm{x}_{1}^{\mathrm{j}}\right)^{2}+\left(\mathrm{y}_{2}^{\mathrm{i}}-\mathrm{y}_{1}^{\mathrm{j}}\right)^{2}}$

After the tests, some exceptions are founded that which the shape $^{\mathrm{i}}$ and shape $\mathrm{j}^{\mathrm{j}}$ are not completely similar although $D_{i, j}^{1} \cong$ $D_{i, j}^{2}$ or $D_{i, j}^{3} \cong D_{i, j}^{4}$.

A solution of this new problem consists on computing the distance using Eq. (5) and Eq. (6) present in Table V.

$D_{i, j}^{5}=\sqrt{\left(x_{c}^{i}-x_{1}^{i}\right)^{2}+\left(y_{c}^{i}-y_{1}^{i}\right)^{2}}$
$D_{i, j}^{6}=\sqrt{\left(\mathrm{x}_{\mathrm{c}}^{\mathrm{j}}-\mathrm{x}_{1}^{\mathrm{j}}\right)^{2}+\left(\mathrm{y}_{\mathrm{c}}^{\mathrm{j}}-\mathrm{y}_{1}^{\mathrm{j}}\right)^{2}}$
Where

$x_{c}^{i}=\left\{x_{1}^{i}+x_{2}^{i}\right\} / 2$

$y_{c}^{i}=\left\{y_{1}^{i}+y_{2}^{i}\right\} / 2$

$x_{c}^{j}=\left\{x_{1}^{j}+x_{2}^{j}\right\} / 2$

$y_{c}^{j}=\left\{y_{1}^{j}+y_{2}^{j}\right\} / 2$

TABLE. IV. LIST OF THE Distances $D_{i, j}^{3}$ AND $D_{i, j}^{4}$ FOR THE SHAPES

\begin{tabular}{|c|c|c|c|c|}
\hline \multirow{2}{*}{ shapes } & \multicolumn{4}{|c|}{ Distances $D_{i, j}^{3}$ and $D_{i, j}^{4}$} \\
\hline & Shape $^{\mathrm{i}}$ & Shape $^{j}$ & $D_{i, j}^{3}$ & $D_{i, j}^{4}$ \\
\hline Shape 1 & 1 & 1 & 87.68 & 87.68 \\
\hline Shape 2 & 1 & 5 & 371.21 & 371.21 \\
\hline Shape 3 & 1 & 27 & 371.21 & 249.81 \\
\hline Shape 4 & 1 & 28 & 517.6 & 342.23 \\
\hline Shape 5 & 2 & 2 & 10.81 & 10.81 \\
\hline Shape 6 & 2 & 4 & 209.11 & 191.09 \\
\hline Shape 7 & 2 & 6 & 52.77 & 40.79 \\
\hline Shape 8 & 2 & 7 & 215.37 & 195.14 \\
\hline Shape 9 & 2 & 8 & 300.85 & 282.33 \\
\hline Shape 10 & 2 & 9 & 113.07 & 113.56 \\
\hline Shape 11 & 2 & 10 & 91.76 & 83.72 \\
\hline Shape 12 & 2 & 14 & 257.50 & 238.44 \\
\hline Shape 13 & 2 & 23 & 295.89 & 285.57 \\
\hline Shape 14 & 2 & 24 & 288.17 & 275.22 \\
\hline Shape 15 & 2 & 25 & 375.41 & 354.27 \\
\hline Shape 16 & 2 & 26 & 406.58 & 384.66 \\
\hline Shape 17 & 2 & 29 & 320.15 & 308.10 \\
\hline Shape 18 & 2 & 31 & 382.20 & 362.94 \\
\hline Shape 19 & 2 & 32 & 366.13 & 353.09 \\
\hline Shape 20 & 2 & 33 & 421.96 & 402.71 \\
\hline Shape 21 & 3 & 3 & 172.04 & 172.04 \\
\hline Shape 22 & 3 & 15 & 268.50 & 269.4 \\
\hline Shape 23 & 3 & 16 & 374.76 & 69.29 \\
\hline Shape 24 & 3 & 30 & 401.06 & 309.09 \\
\hline Shape 25 & 11 & 11 & 119.50 & 119.50 \\
\hline Shape 26 & 11 & 13 & 224.32 & 93.52 \\
\hline Shape 27 & 11 & 20 & 224.32 & 92.19 \\
\hline Shape 28 & 11 & 21 & 294.15 & 55.86 \\
\hline Shape 29 & 12 & 12 & 64.84 & 64.84 \\
\hline Shape 30 & 12 & 18 & 140.73 & 140.73 \\
\hline Shape 31 & 12 & 19 & 188.09 & 65.05 \\
\hline Shape 32 & 12 & 22 & 215.93 & 160.82 \\
\hline Shape 33 & 17 & 17 & 101.82 & 101.82 \\
\hline
\end{tabular}


TABLE. V. List OF the Distances $D_{i, j}^{5}$ AND $D_{i, j}^{6}$ FOR THE ShaPeS

\begin{tabular}{|c|c|c|c|c|}
\hline \multirow{2}{*}{ shapes } & \multicolumn{4}{|c|}{ Distances $D_{i, j}^{5}$ and $D_{i, j}^{6}$} \\
\hline & Shape $^{\mathrm{i}}$ & Shape $^{j}$ & $D_{i, j}^{5}$ & $D_{i, j}^{6}$ \\
\hline Shape 1 & 1 & 1 & 43.84 & 43.84 \\
\hline Shape 2 & 1 & 5 & 43.84 & 43.84 \\
\hline Shape 3 & 1 & 27 & 43.84 & 43.84 \\
\hline Shape 4 & 1 & 28 & 43.84 & 43.84 \\
\hline Shape 5 & 2 & 2 & 5.40 & 5.40 \\
\hline Shape 6 & 2 & 4 & 5.40 & 5.40 \\
\hline Shape 7 & 2 & 6 & 5.40 & 5.0 \\
\hline Shape 8 & 2 & 7 & 5.40 & 5.40 \\
\hline Shape 9 & 2 & 8 & 5.40 & 5.40 \\
\hline Shape 10 & 2 & 9 & 5.40 & 5.70 \\
\hline Shape 11 & 2 & 10 & 5.40 & 5.40 \\
\hline Shape 12 & 2 & 14 & 5.40 & 5.40 \\
\hline Shape 13 & 2 & 23 & 5.40 & 5.40 \\
\hline Shape 14 & 2 & 24 & 5.40 & 5.70 \\
\hline Shape 15 & 2 & 25 & 5.40 & 5.40 \\
\hline Shape 16 & 2 & 26 & 5.40 & 5.70 \\
\hline Shape 17 & 2 & 29 & 5.40 & 5.40 \\
\hline Shape 18 & 2 & 31 & 5.40 & 5.00 \\
\hline Shape 19 & 2 & 32 & 5.40 & 5.70 \\
\hline Shape 20 & 2 & 33 & 5.40 & 5.40 \\
\hline Shape 21 & 3 & 3 & 86.02 & 86.02 \\
\hline Shape 22 & 3 & 15 & 86.02 & 86.02 \\
\hline Shape 23 & 3 & 16 & 86.02 & 86.54 \\
\hline Shape 24 & 3 & 30 & 86.02 & 86.02 \\
\hline Shape 25 & 11 & 11 & 59.75 & 59.75 \\
\hline Shape 26 & 11 & 13 & 59.75 & 59.75 \\
\hline Shape 27 & 11 & 20 & 59.75 & 59.75 \\
\hline Shape 28 & 11 & 21 & 59.75 & 59.39 \\
\hline Shape 29 & 12 & 12 & 32.42 & 32.42 \\
\hline Shape 30 & 12 & 18 & 32.42 & 32.42 \\
\hline Shape 31 & 12 & 19 & 32.42 & 32.42 \\
\hline Shape 32 & 12 & 22 & 32.42 & 32.42 \\
\hline Shape 33 & 17 & 17 & 50.91 & 50.91 \\
\hline
\end{tabular}

Now if

$$
\left(D_{i, j}^{1} \cong D_{i, j}^{2} \text { and } D_{i, j}^{5} \cong D_{i, j}^{6}\right) \text { or }\left(D_{i, j}^{3} \cong D_{i, j}^{4} \text { and } D_{i, j}^{5} \cong D_{i, j}^{6}\right)
$$

Shapei and shapej are very similar.

\section{B. Family of Similar Shapes in the Islamic Pattern}

In this paper the approach, after having extracted the similar regions [10-11], a problem related to similar regions is founded. For example, if they are three regions, $F_{1}$ with index $1, F_{2}$ with index 6 and $F_{3}$ with index 27 , the program will show that $F_{1}$ is similar to $F_{1}, F_{1}$ is similar to $F_{2}, F_{1}$ is similar to $F_{3}, F_{2}$ is similar to $F_{2}, F_{2}$ is similar to $F_{3}$ and $F_{3}$ is similar to $F_{3}$.

The objective is to eliminate the combinations $F_{2}$ is similar to $F_{2}, F_{2}$ is similar to $F_{3}$ and $F_{3}$ is similar to $F_{3}$.
To solve this problem, a set of points created, and testing whether the shape is already present in the set. If that it is so, then it not inserted in the set. Else, it will be insert it in the set of points. Table III shows the results with this method of computing the Euclidian distance among different regions.

In this subsection, which display the different family of geometric shapes, and the numbers of all shapes composing the family. Table VI shows results of different shapes in Hasba Pictures.

\section{Units Display of the different Family Groups}

In this subsection, witch display the results obtained through using this new proposed approach for the detection of the similar geometric shapes in Islamic geometric patterns or Hasba Pictures Fig. 16.

TABLE. VI. FAMILY OF EACH SHAPE

\begin{tabular}{|l|l|l|}
\hline \multirow{2}{*}{ Family's } & \multicolumn{2}{|l|}{ Number of shapes for each family } \\
\cline { 2 - 3 } & family shapes & Number \\
\hline Family 1 & $1,5,27,28$ & 4 \\
\hline Family 2 & $\begin{array}{l}2,4,6,7,8,9,10,14,23, \\
24,25,26,29,31,32,33\end{array}$ & 16 \\
\hline Family 3 & $3,15,16,30$ & 4 \\
\hline Family 4 & $1911,13,20,21$ & 4 \\
\hline Family 5 & $12,18,19,22$ & 4 \\
\hline Family 6 & 17 & 1 \\
\hline
\end{tabular}
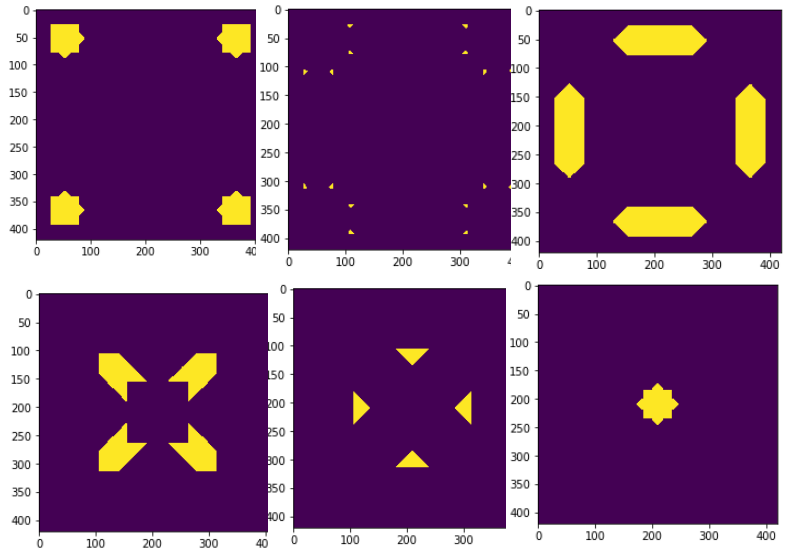

Fig. 16. Family Group of Shapes.

\section{CONCLUSIONS}

Many works have used the simple blob detection method to show us the different connected components in a binary picture. The problem treated in this work is not only the detection of connected components but also how to extract the similar regions in an Islamic geometric pattern. This paper presented a new approach to solve the problem: How can extract a family group of each shape in the pictures constructed with the method called 'Hasba'. In a future work, the objective is to implement new moments and apply the new detected approach to know whether a given kind of Islamic geometric patterns is valid or not. 


\section{ACKNOWLEDGMENT}

The authors are grateful to Prof. Dr. Thalal Abdelmalek for pictures from Hasba presented in this paper and for fruitful discussions.

\section{REFERENCES}

[1] Thalal, A., Benatia, M. J., Jali, A., Aboufadil, Y. \& Elidrissi Raghni, M. A. (2011). Symmetry Culture Sci. 22, 1-2, 103-130.

[2] Aboufadil Y, Thalal A, Elidrissi Raghni MA. Moroccan ornamental quasiperiodic patterns constructed by the multigrid method. J Appl Cryst. 2014; 47:630-641.

[3] http://www.labbookpages.co.uk/software/imgProc/blobDetection.html.

[4] https://en.wikipedia.org/wiki/Euclidean_distance.

[5] https://en.wikipedia.org/wiki/Connected-component_labeling.

[6] Azami N., Idrissi D.E., Amrane S., Harmouchi M. Computer blob detection and tracking for highly repeatable optical fiber sensor; Proceedings of the 2014 9th International Conference on Intelligent Systems: Theories and Applications (SITA-14); Rabat, Morocco. 7-8 May 2014; pp. 1-5.

[7] Philipp S., Vieira B., and Sanfourche M., "Fuzzy Segmentation of Color Images and Indexing of Fuzzy Regions," in proceedings of Conference on Colour in Graphics, Imaging, and Vision, Poitiers, pp. 507-512, 2002.

[8] Eum S., Jung H.G. Enhancing Light Blob Detection for Intelligent Headlight Control Using Lane Detection. IEEE Trans. Intell. Transp. Syst. 2013;14:1003-1011. doi: 10.1109/TITS.2012.2233736.

[9] Patro B.N. Design and implementation of novel image segmentation and BLOB detection algorithm for real-time video surveillance using DaVinci processor; Proceedings of the 2014 International Conference on Advances in Computing, Communications and Informatics (ICACCI); Delhi, India. 24-27 September 2014; pp. 1909-1915.

[10] Wu K., Otoo E.J., Suzuki K. Optimizing two-pass connected-component labeling algorithms. Pattern Anal. Appl. 2009;12:117-135. doi: 10.1007/s10044-008-0109-y.

[11] Kiran B., Ramakrishnan K., Kumar Y., Anoop K.P. An improved connected component labeling by recursive label propagation; Proceedings of the 2011 National Conference on Communications (NCC); Bangalore, India. 28-30 January 2011; pp. 1-5.

[12] Derrode S. and Ghorbel F., "Robust and Efficient Fourier-mellin Transform Approximations for Invariant Grey-level Image Description and Reconstruction," Computer Vision and Image Understanding, vol. 83, no. 1, pp. 57-78, 2001.

[13] Chang F., jen Chen C., jen Lu C. A linear-time component-labeling algorithm using contour tracing technique. Comput. Vis. Image Underst. 2004;93:206-220. doi: 10.1016/j.cviu.2003.09.002.

[14] T. Q. CHEN, Y. LU, Color image segmentation: an innovative approach, Pattern Recognition 35 (2), 395-405, (2002).

[15] Nguyen T.B., Chung S.T. An Improved Real-Time Blob Detection for Visual Surveillance; Proceedings of the CISP '09. 2nd International Congress on Image and Signal Processing; Tianjin, China. 17-19 October 2009; pp. 1-5.

[16] Y. DENG, B. MANJUNATH, Unsupervised segmentation of colortexture regions in images and video, IEEE Trans. on Pattern Analysis and Machine Intelligence 23 (8), 800-810, (2011).

[17] Sameer A., Rangachar K., and Ramesh J., "A Survey on the Use of Pattern Recognition Methods for Abstraction, Indexing and Retrieval of Images and Video," Pattern Recognition, vol. 35, no. 4, pp. 945-965, 2002 .

[18] https://fr.scribd.com/document/97422644/07-Pictures-Binaires.

\section{NOTES ON CONTRIBUTORS}

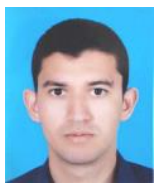

Ait Lahcen Yassine is currently a PhD student at Cadi Ayyad University, Marrakesh, Morocco, with the main field of interest being Computer Science and other fields of interest being Moroccan Art. The author's current research interest is computational method for Moroccan geometric art, digital construction of geometric patterns and modelling. The author's research activities are three Communications in the 2 last years.

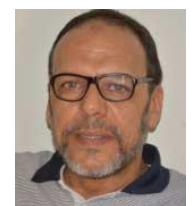

Jali Abdelaziz is Ph.D. of University Higher Normal School Paris, University Paris 13, with the main field of interest being Numerical Analysis and other fields of interest being Mathematics current research interest is computational method for Moroccan geometric art, digital construction of Computer programming infography. The author's current research interest is Moroccan Art and Computer programming. The author is Assistant Professor, Department of Mathematics, Cadi Ayyad University, Marrakesh, and Member of the Council of the Department of Mathematics, University Cadi Ayyad, and Marrakesh. The author's research activities are 7 Publications and Communications in the 10 last years, Supervision of 2 Theses, and Supervision of 12 Master's projects.

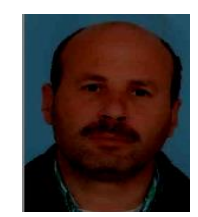

El Oirrak Ahmed joined Cadi Ayyad University, Morocco, in 1999, first as an assistant professor, and received the Doctorate and Habilitation in signal processing from the Mohammed V University, Morocco, in 2001 and University Cadi Ayyad, Morocco, in 2010 respectively. He is presently a PES professor with the Faculty of Sciences of Marrakech Semlalia. His research interests include image processing, pattern recognition and their applications. He is the author or coauthor of more than 20 publications.

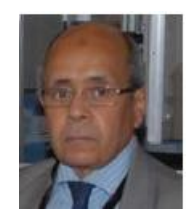

Thalal Abdelmalek is a Doctorat d'Etat $(\mathrm{PhD})$ in Materials Sciences, P\&M Curie University, Paris 6, and France. The author's main field of interest is Materials Sciences. Other fields of interest include Physics and the current research interest is Moroccan Art and Crystallography. The author is Professor, Department of Physics, Cadi Ayyad University, and Marrakech. Chairperson of the 24th European Crystallographic Meeting Conference (ECM24)-2007. President of the Moroccan Crystallographic Association (AMC), Organizer of 6 Moroccan Schools of Crystallography, Coordinator of the International Year of Crystallography Activities in Morocco: OpenLabs Morocco-2014, Chairman of the conference "Crystallography for the next generation: the legacy of IYCr" -Rabat-2015,Member of the Scientific Board of the International Basic Sciences Programme (IBSP) -UNESCO. The author's research activities include 20 Selected Publications and Communications in the 10 last years and Supervision of 10 Theses.

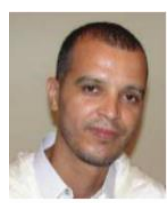

Aboufadil Youssef is Doctorat (PhD) in Crystallography, Cadi Ayyad University, Marrakesh, Morocco, with the main field of interest being Materials Science. Other fields of interest include Physics. The author's current research interest is Crystallography, Ceramic and Moroccan Art. The author is Assistant Professor, Department of Physics, Cadi Ayyad University, and Marrakesh. Member of the Moroccan Crystallographic Association (AMC), participant in the organization of the 24th European Crystallographic Meeting Conference (ECM24) - 2007, participant in the organization of the conference "Crystallography for the next generation: the legacy of IYCr" - Rabat - 2015 The author's research activities are 18 Publications and Communications in the 4 last years and 6 workshops in Symmetry and Geometric Art.

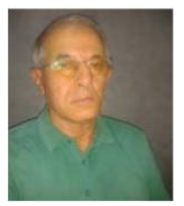

El Idrissi Raghni My Ahmed is Doctorat d'Etat $(\mathrm{PhD})$ in Materials Sciences,Cadi Ayyad university, Marrakesh Marocco, with the main field of interest beingMaterials Science. Other fields of interest include Physics and the current research interest is Moroccan Art and Ceramic Pigment. The author is Professor, Department of Physics, Cadi Ayyad University,Marrakesh, Director of the Laboratory of Materials Sciences, Faculty of Sciences Semlalia, Cadi Ayya University, participant in the organization of the 24th European Crystallographic Meeting Conference (ECM24) - 2007. The author's research activities are 15 Publications in the 10 last years and Supervision of 4 Theses. 